\title{
EDITORIAL
}

\section{POBLACIÓN INMIGRANTE Y ENFERMEDADES INFECCIOSAS}

\author{
Antonio Guerrero Espejo y Javier Colomina Rodríguez \\ Servicio de Microbiología (Unidad de Investigación en Patología Infecciosa). Área de Diagnóstico Biológico. Hospi- \\ tal de la Ribera, Alzira (Valencia).
}

El fenómeno de la inmigración se ha intensificado en España durante la última década y su crecimiento ha sido exponencial en los últimos años. En este año nos podemos aproximar a los dos millones de inmigrantes de los cuales medio millón viven en una situación administrativa irregular.

En la Unión Europea se calcula que viven en situación ilegal y han entrado clandestinamente unos 4 ó 5 millones de personas procedentes del norte de África, Oriente Próximo, Europa del Este, América Latina e India. De acuerdo con los datos del Ministerio del Interior en su anuario estadístico de extranjería del 2002, los extranjeros con permiso de residencia en vigor en España a 31 de diciembre de 2002 eran de 1.324 .000 personas $^{1}$. De acuerdo al continente de origen, el $35 \%$ de los extranjeros son europeos, el $28 \%$ africanos, el $29 \%$ latinoamericanos y el $8 \%$ asiáticos. Según nacionalidad, los colectivos mayoritarios son el marroquí (282.432 personas), el ecuatoriano (115.301), el británico (90.091), el colombiano (71.238) y el alemán (65.823). Hasta el $80 \%$ de los extranjeros en España se agrupan en unas pocas Comunidades Autónomas (Cataluña con 328.461 extranjeros con

Correspondencia:

Antonio Guerrero Espejo.

Área de Diagnóstico Biológico

Hospital de La Ribera

Ctra. Corbera Km 1, 46600-Alzira (Valencia)

Correo electrónico: aguerrero@ hospital-ribera.com permiso de residencia, Madrid con 272.692, Andalucía con 163.942, la Comunidad Valenciana con 138.421 , Canarias con 92.149 y las Islas Baleares con 60.779). No existen grandes diferencias por sexo ya que, según los datos actuales, el $45 \%$ son mujeres y el $55 \%$ son varones.

Por otro lado, a diferencia de lo que pudiera pensarse, las Naciones Unidas estiman que a mitad del siglo actual, se necesitarán más de 50 millones de inmigrantes para mantener el sistema de pensiones y el estado de bienestar en la Unión Europea. Al parecer, nuestro país debería recibir cada año unos 250.000 inmigrantes para mantener el crecimiento económico.

\section{ENFERMEDADES QUE AFECTAN A LOS INMIGRANTES}

En España los problemas de salud de las poblaciones inmigradas son similares a los de la población autóctona ${ }^{2}$, predominando en el ámbito de la atención primaria las infecciones respiratorias, los síndromes depresivos y los dolores lumbares ${ }^{3}$. Entre los adolescentes, por ejemplo, prevalecen las enfermedades odontológicas, respiratorias y cutáneas como consecuencia de infecciones o ausencia de adecuada higiene ${ }^{4}$.

La perspectiva de las enfermedades que afectan a los inmigrantes varía según la 
población atendida y el nivel asistencial (atención primaria versus atención especializada) ${ }^{5}$. Así en una unidad de referencia de medicina tropical con atención mayoritaria de inmigrantes subsaharianos indocumentados, la fiebre, el prurito, la eosinofilia, la visceromegalia y la anemia fueron más frecuentes entre los africanos, mientras que la tos lo fue entre los latinoamericanos ${ }^{6}$. Los diagnósticos más frecuentes fueron filariasis, malaria, parasitaciones intestinales, infección por los virus de hepatitis $\mathrm{C}$ y B, enfermedades de transmisión sexual, tuberculosis e infección por el $\mathrm{VIH}^{5}$.

Aunque las tasas de infección por el VIH en poblaciones del África subsahariana son elevadas, la incidencia del VIH en España es superior a la de determinados países de Sudamérica y África del Norte de donde procede un alto porcentaje de inmigrantes de nuestro país ${ }^{7}$. Hasta el año 2000, se han notificado en España más de 1.000 casos de sida en inmigrantes (menos del 3\% del total de casos), lo que demuestra que la epidemia de infección por VIH es autóctona en España y no está condicionada por la inmigración. La población inmigrante es, por el contrario, altamente vulnerable a la infección, debido al uso de la prostitución de bajo nivel social en colectivos de inmigrantes masculinos y al ejercicio de la misma al que se ven abocadas/os muchos de ellos.

\section{LOS PROFESIONALES DE LA SALUD Y ATENCIÓN SANITARIA A LOS INMIGRANTES}

Al igual que ocurre en muchos países entre la población general, los profesionales sanitarios estamos mal preparados para adaptarnos a los rápidos cambios demográficos de la inmigración y la actitud hacia el movimiento migratorio se puede estar endureciendo $0^{8,9}$. La atención sanitaria de las personas inmigrantes despierta preocupación y controversia política y social. Se ha considerado por algunos, que los inmigrantes supo- nen una amenaza para la salud y esta idea da pie a sentimientos xenófobos.

Los patrones de utilización, incluyendo el uso abusivo de los servicios sanitarios por los inmigrantes, que varían con el tiempo de estancia en el país ${ }^{10}$, constituyen una de las quejas de determinados trabajadores sanitarios.

En general los profesionales sanitarios no tenemos formación suficiente para entender cómo la cultura, la raza y otros factores étnicos afectan a la aceptación por parte del paciente de las prácticas sanitarias tradicionales de occidente. Es una realidad que, en ocasiones, las diferencias entre sanitarios y pacientes crean a veces barreras y situaciones tensas durante la atención sanitaria ${ }^{11,12}$.

Además, la comunicación no es fácil entre miembros de grupos étnicos y sanitarios a causa de las barreras lingüísticas o el nivel cultural que condiciona la expresión de los síntomas. Las diferencias culturales afectan también en ocasiones a la aportación de datos en la historia clínica. Por ejemplo, la hematuria en los niños egipcios debido a esquistosomiasis vesical es tan común que se considera normal en el delta del Nilo, al norte del Cairo. Existe la transmisión potencial de infecciones como la hepatitis B o el VIH a través de agujas no esterilizadas usadas para la acupuntura $\mathrm{u}$ otras inyecciones realizadas por sanadores tradicionales. Esta vía adquiere importancia al considerar que la prevalencia de infecciones por virus hepatotropos pueden ser altísimas en colectivos como los inmigrantes subsaharianos ${ }^{13}$.

\section{IMPACTO DE LAS ENFERMEDADES INFECCIOSAS EN LA SALUD PÚBLICA}

La mayoría de las enfermedades infecciosas típicas de los inmigrantes no tienen impacto, en términos de salud pública, en el país de destino puesto que su transmisión es 
muy difícil en nuestro medio, ya sea por la falta del vector o de los hospedadores intermediarios ${ }^{14}$. No obstante, la tuberculosis, por su frecuencia e impacto en la salud pública merece una especial consideración. En general, desde el punto de vista de la tuberculosis la inmigración no supone un riesgo grave para la salud pública del país de acogida ya que, aunque la incidencia de tuberculosis en algunos de estos colectivos es superior a la de la población española, las tasas de tuberculosis en nuestro país continúan descendiendo y no se han visto claramente afectadas por la inmigración ${ }^{15,16}$. No obstante, la disminución de la tuberculosis en algunos países de Europa occidental se ha detenido principalmente debido a la inmigración procedente de países con alta prevalencia $^{17}$.

La situación de algunos países europeos, en los que más de la mitad de los casos de tuberculosis diagnosticados son inmigrantes, no se da en España ${ }^{18}$. No obstante, en los últimos años en Barcelona el porcentaje de inmigrantes entre los enfermos de tuberculosis ha aumentado hasta el $32 \%$, con una tasa entre inmigrantes que alcanza hasta 100 casos/100.000 habitantes/año ${ }^{19}$. Esto hace que la población inmigrante siga siendo un grupo prioritario para la vigilancia y control de la tuberculosis. También es importante destacar que la tuberculosis importada en países industrializados y que se presenta en brotes epidémicos no es transmitida sólo por inmigrantes (condicionadas por las condiciones de vida de hacinamiento, pobreza, etc.), sino también por otros colectivos que han visitado países con alta incidencia de la enfermedad ${ }^{20}$.

La situación irregular y las condiciones higiénico-sanitarias de vida suponen el verdadero riesgo para la salud de los inmigrantes, respecto a determinadas patologías como la tuberculosis ${ }^{21}$. La transmisión de tuberculosis entre ellos es fácil de comprender, si tenemos en cuenta que la mayoría de las familias llegan a vivir en casas con una media de 2,3 personas por habitación y que el $88 \%$ de las mismas comparten casa ${ }^{22}$.

La infección tuberculosa latente es muy variable según el área geográfica de procedencia en un mismo continente, de tal manera que entre inmigrantes recién llegados las tasas son muy variables como se aprecia en el artículo de Alonso y colaboradores, publicado en este mismo número ${ }^{23-27}$. Con frecuencia, una induración superior a $10 \mathrm{~mm}$ en la prueba de Mantoux, se atribuye a la vacunación en la infancia con el bacilo CalmeteGuérin, usado en algunos países donde el riesgo de tuberculosis es elevado. Sin embargo, en la mayoría de las ocasiones, esa prueba positiva refleja una infección subclínica y predice el riesgo de infección de enfermedad activa en el futuro, por lo que las personas con intradermoreacción positiva deben ser evaluadas para excluir enfermedad activa.

La idea de que en un país con baja incidencia general la incidencia de la tuberculosis en inmigrantes se reduce con rapidez durante los primeros años de estancia no es cierta en determinados colectivos de inmigrantes $^{21}$. La investigación de los contactos cercanos de inmigrantes con tuberculosis es muy coste-efectiva para la salud pública ${ }^{28}$.

\section{RETO DIAGNÓSTICO DE ENFERMEDADES TROPICALES}

Sin lugar a duda, la inmigración supone un reto para los clínicos, ya que deben de incluir en su diagnostico diferencial enfermedades endémicas de las áreas de procedencia $^{29}$. Es bien conocido que algunas patologías emergentes y/o re-emergentes son derivadas de la movilidad internacional de la población. En España, han aparecido en los últimos 25 años más de 22 procesos asociados a la inmigración, algunos de los cuales ya estaban erradicados.

Así, los inmigrantes recién llegados o que llevan poco tiempo en nuestro país pueden 
padecer un gran número y variedad de infecciones, pero con el paso de los años de estancia en España la posibilidad de padecer enfermedades propias de su país de origen se reduce de forma significativa. Por el contrario, el riesgo para otras infecciones como la tuberculosis, la estrongiloidiasis, esquistosomiasis o la equinococosis, puede persistir durante el resto de su vida.

En la evaluación de las personas que vienen de otros países hay que considerar datos especiales. Por ejemplo la inmunidad suele estar presente frente a virus como el de la hepatitis A o el virus de Epstein-Barr, que suele infectar a los niños en los países en desarrollo. Las políticas de vacunación también varían entre países o incluso de unas zonas geográficas a otras, y las personas que han pasado su infancia en países en vías de desarrollo, con frecuencia no han recibido las vacunaciones que se dan rutinariamente en España. Por ejemplo, en muchas áreas, nunca recibieron la inmunización primaria para el tétanos, hepatitis B, Haemophilus influenzae, Neisseria meningitidis tipo C, etc.

Algunos datos clínicos o de laboratorio también deben ser evaluados específicamente en el caso de las personas inmigrantes. Así, la presencia de anemia y/o eosinofilia sugiere la posibilidad de parasitosis intestinal que en el inmigrante con frecuencia es poliparasitaria. Por otro lado, la prueba serológica frente a la sífilis es positiva a veces reflejando la infección con otras treponematosis de zonas endémicas, como el Bejel o el Pian.

En la población inmigrante pueden estar presentes otras infecciones latentes o crónicas, además de la tuberculosis, que no le son familiares al clínico, como el paludismo, estrongiloidiasis, tripanosoniasis, esquistosomiasis o filariasis, por citar las más comunes. Por ejemplo, pocos profesionales considerarían que un paciente con paludismo esté asintomático en el momento del diagnós- tico $^{30}$. Además, algunas de las infecciones crónicas tienen secuelas inesperadas, como la hepatitis B que se asocia con cáncer hepático, Schistosoma haematobium con cáncer de vesícula o Trypanosoma cruzi (enfermedad de Chagas) con defectos de conducción y dilatación del tracto gastrointestinal o del uréter.

Determinadas complicaciones, poco comunes en España, pueden también pasar desapercibidas. Así, la infección recurrente del tracto urinario se origina a veces por secuelas de una tuberculosis génito-urinaria silente. Un gusano helminto que realiza migración intraorgánica como Ascaris lumbricoides puede provocar colangitis aguda. La infiltración pulmonar cavitada con un Mantoux positivo y hemoptisis puede ser ocasionada por Paragonimiasis (infección por el trematodo pulmonar Paragonimus westermani). La neurocisticercosis, frecuente en inmigrantes de Latinoamérica, es una causa de crisis convulsivas que tampoco debe olvidarse ${ }^{31}$.

En este número de la revista, Carrillo y colaboradores publican un interesante artículo en el que plantean la necesidad de realizar una prueba de cribado para la detección de una patología emergente en nuestro país como la loasis (infección por la filaria Loa loa $)^{32}$.

En definitiva, los profesionales sanitarios debemos saber afrontar esta nueva realidad que supone la inmigración en el contexto sanitario. Los problemas de salud que presenten los inmigrantes, el control de algunas enfermedades con potencial epidémico para la sociedad española o el diagnóstico precoz de enfermedades infecciosas emergentes son las principales demandas que se nos plantean actualmente. La realidad de otros países europeos que nos han precedido en la experiencia de la inmigración, puede ayudarnos a superar prejuicios innecesarios y a establecer mejores programas de vigilancia y control. 


\section{BIBLIOGRAFÍA}

1. Delegación del Gobierno para la Extranjería y la Inmigración. Observatorio permanente de la inmigración. Anuario estadístico de extranjería 2002. Consultado 15-08-2004. Disponible en: http:// www.mir.es/dgei.

2. Sanz B, Torres A, Schumacher R. Características sociodemográficas y utilización de servicios sanitarios por la población inmigrante residente en un área de la Comunidad de Madrid. Aten Primaria 2000; 26: 314-18

3. Pena M. Motivos de consulta y características demográficas de una comunidad de inmigrantes «sin papeles» en el distrito de Usera-Villaverde (Madrid). Aten Primaria 2001; 27: 25-8.

4. Olivan G. Evaluación del estado de salud y nutrición de los adolescentes inmigrantes ilegales de origen magrebí. An Esp Pediatr 2000; 53: 17-20.

5. Roca C, Balanzo X, Fernandez-Roure JL, Sauca G, Savall R, Gascón J, et al. Enfermedades importadas en inmigrantes africanos: estudio de 1.321 pacientes. Med Clin (Barc) 2002; 119: 616-9.

6. López-Vélez R, Huerga H, Turrientes MC. Infectious diseases in immigrants from the perspective of a tropical medicine referral unit. Am J Trop Med Hyg 2003; 69: 115-21.

7. Llácer A, Del Amo J, Castollo S, Belza MJ. Salud e inmigración: a propósito del Sida. Gac Sanit 2001; 15: 197-9.

8. Carballo M, Nerukar A. Migration, refugees, and health risks. Emerg Infect Dis 2001; 7 (3 Suppl): 556-60.

9. Martín LM. El paciente inmigrante en atención primaria. ¿Estamos preparados? Aten Primaria 2001; 28: 89-90.

10. Leduc N, Proulx M. Patterns of health services utilization by recent immigrants. J Immigr Health 2004; 6: 15-27.

11. Nuñez GR. Culture, demographics, and critical care issues: an overview. Crit Care Clin 2003; 19: 619-6vi.

12. Documet PI, Sharma RK. Latinos' health care access: financial and cultural barriers. J Immigr Health 2004; 6: 5-13.

13. López-Vélez R, Turrientes C, Gutiérrez C, Mateos $\mathrm{M}$. Prevalence of hepatitis B, C and D markers in sub-saharian African immigrants. J Clin Gastroenterol 1997; 25: 650-2.
14. Gascón J. Enfermedades infecciosas e inmigración. Enferm Infecc Microbiol Clin 2003; 21: 535-9.

15. Vieira Pascual MC, Bischofberger C, Pérez de Oteyza C. Estudio epidemiológico y clínico de los pacientes diagnosticados de tuberculosis en el área noroeste de Madrid. An Med Interna 2003; 20: 10 5 .

16. Surveillance of tuberculosis in Europe-EuroTB Report on tuberculosis cases notified in 2001. European Comisión. Consultado 12-08-2004. Disponible en http://www.eurotb.org

17. Heldal E, Dahle UR, Sandven P, Caugant DA, Brattaas N, Waaler HT, et al. Risk factors for recent transmission of Mycobacterium tuberculosis. Eur Respir J 2003; 22: 637-42.

18. Carballo M, Divino JJ, Zeric D. Migration and health in the European Union. Trop Med Int Health 1998; 3: 936-44.

19. Balagué M, Orcau A, Sánchez P, Tortajada C, Caylà J. Epidemiología actual de la tuberculosis en España: hacia una mejor vigilancia y control. Control Calidad SEIMC. Disponible en: www. seimc.org/control/revi_Micobac/Epibc.htm.

20. Valles X, Sánchez F, Pañella H, García de Olalla P, Jansà J, Caylà J. Tuberculosis importada: una enfermedad emergente entre países industrializados. Med Clin (Barc) 2002; 188: 376-8.

21. Lillebaek T, Andersen AB, Dirksen A, Smith E, Skovgaard LT, Kok-Jensen A. Persistent high incidence of tuberculosis in immigrants in a low-incidence country. Emerg Infect Dis 2002; 8: 679-84.

22. Benítez RT, Llerena AP, López GR, Brugera MC, Lasheras L. Determinantes socioeconómicos en un colectivo de familias inmigrantes. An Pediatr (Barc ) $2004 ; 60: 9-15$.

23. Alonso FJ, García MC, Lougedo MJ, Comas JM, García M, López F, Pérez N, Sánchez R, Soto M. Prevalencia de infección tuberculosa en las personas inmigrantes del área de salud de Toledo. Rev Esp Salud Pública 2004; 78: 593-600.

24. Pérez-Arellano JL, Hernández-García A, SanzPeláez AA. Inmigración africana en Canarias e infección tuberculosa. Med Clin (Barc) 2002; 118: 38 .

25. Durán E, Cabezos J, Ros M, Terre M, Zarzuela F, Bada JL. Tuberculosis en inmigrantes recién llegados a Barcelona. Med Clin (Barc) 1999; 6: 525-8.

26. Rivas FJ, Nácher M, Corrillero J, García-Herreros MT. Prevalencia de infección tuberculosa entre los 
inmigrantes magrebíes. Med Clin (Barc) 2000; 114: $245-9$.

27. Ramos JM, Pastor C, Masía MM, Chascales E, Royo G, Gutiérrez E. Examen de salud en la población inmigrante: prevalencia de infección tuberculosa latente, hepatitis $\mathrm{B}$, hepatitis $\mathrm{C}$, virus de inmunodeficiencia humana y sífilis. Enf Inf Microbiol Clin 2003; 21: 540.2 .

28. Dasgupta K, Schwartzman K, Marchand R, Tennenbaum TN, Brassard P, Menzies D. Comparison of cost-effectiveness of tuberculosis screening of close contacts and foreign-born populations. Am J Respir Crit Care Med 2000; 162: 2079-86.
29. Worley CW, Worley KA, Kumar PL. Infectious disease challenges in immigrants from tropical countries. Pediatrics 2000; 106: E3.

30. Cabezos J, Durán E, Treviño B, Bada JL. Paludismo importado por inmigrantes en Cataluña . Med Clin (Barc) 1995; 104:45-8.

31. Roca C, Gascón J, Font B, Pujol T, Valls ME, Corachán M. Neurocysticercosis and population movements analysis of 23 imported casas in Spain. Eur J Clin Microbiol Infect Dis 2003; 22: 382-4.

32. Carrillo E, Iglesias B, Gómez J, Guinovart C, Cabezos J. Cribaje de microfilariasis sanguínea (Loa loa) en la población de Barcelona inmigrante de zonas endémicas. Rev Esp Salud Pública 2004; 78: 623-30. 